\section{ekf}

VYSOKÁ ŠKOLA BÁŇSKÁ EKONOMICKÁ FAKULTA

\title{
Fuzzy weights estimation method based on the linguistic expression of criteria relevance
}

\author{
Adam BOROVIČKA*
}

Department of Econometrics, Faculty of Informatics and Statistics, University of Economics, W. Churchill Sq. 4, 13067 Prague 3, Czech Republic.

\begin{abstract}
In this article, the quantified expression of criteria weights in decision-making processes is considered. A decision maker is often able to express his or her preferences only linguistically. The concept of fuzzy numbers, or linguistic variables, is used in order to state these vague preferences. A fuzzy number is considered as one with a triangular membership function, which renders an approximate value of the weight of a particular criterion according to linguistic terms about its importance. To set the strict (crisp) value of weights, the optimization model based on the maximin principle is proposed in two modifications. The proposed approaches are compared with other methods. Finally, the introduced methods are applied to a rather illustrative but real case of investment decision making in the capital market. The main results are discussed.
\end{abstract}

\section{Keywords}

Fuzzy number, fuzzy weight, linguistic expression, membership function.

JEL Classification: C44, C61

*adam.borovicka@vse.cz 


\title{
Fuzzy weights estimation method based on the linguistic expression of criteria relevance
}

\author{
Adam BOROVIČKA
}

\section{Introduction}

If we are solving a particular problem via models and methods of decision-making theory, the importance of particular criteria must be quantitatively expressed. In the literature, we can find many approaches working with concrete, quantified information about criterion relevance from the side of the decision maker. These methods can be seen collectively, for instance in Hwang and Yoon (1981), Fiala (2008) or Brožová et al. (2009).

In this article, another situation will be considered, which is more real. Namely, the decision maker states the criterion importance linguistically: thus, very important, less important or something similar. This vague preference is expressed by a triangular fuzzy number in order to describe at least an approximate value of a criterion weight. For this purpose, the concept of linguistic variables is applied (Zadeh, 1975a, b, c). The transformation process of linguistic terms to fuzzy numbers will be described as a rather human intuitive procedure. The inspiration for this process can be found for example in Baas and Kwakernaak (1977), Kerre (1982) or Chen (1988). Finally, to set the final quantitative (crisp) value of weights, a mathematical model will be created that is computationally based on the multiple objective optimization maximin principle (Bellman and Zadeh, 1970; Zimmermann, 1978). It is possible to consider this procedure as a kind of defuzzification process, in which several computational formulae are proposed, e.g. by Chen and Hwang (1992). In this case, however, it is defuzzification with additional conditions. Therefore, the optimization process will be discussed, similar to Lai and Hwang (1996).

The main aim of the article is to solve the situation in which crisp criteria weights are needed, but only a linguistic expression about the criteria relevance is available. To solve this problem, a new methodical procedure is proposed. The proposed method is an alternative to methods using defuzzification formulae, in which subsequent weight normalization must be performed (Chen and Hwang, 1992). Other methods use a composition operator or composite membership function in order to express fuzzy priorities (Narasimhan, 1981). This process can be computationally more difficult. The proposed approach should be userfriendly, not computationally difficult.

In order to understand all the processes, the basic pieces of knowledge of the fuzzy set theory are introduced. The concept of triangular fuzzy numbers, the basic operations with fuzzy sets, the linguistic variables and the fuzzy programming problem are presented.

In order to comprehend the proposed approaches, a real application is provided from the field of the capital market, in which the potential investor needs to make a decision about investing in open share funds.

The structure of the article is as follows. First, the basic notions of the fuzzy set theory, triangular fuzzy numbers, linguistic variables and Bellman's approach are described. In the next chapter, the weight estimation method is proposed. Finally, the method is applied to illustrative investment decision-making situations. The results are discussed and the proposed approach is compared with other methods.

\section{Fuzzy set theory}

We are not always able to express our opinions exactly. Many manners are only vague and uncertain. In order to model these situations more precisely, the modified set theory was developed. This concept is known as fuzzy set theory, proposed by L.A. Zadeh (1965).

\subsection{Basic notions}

Let us briefly explain the basic principle of the concept mentioned above. Similar to Dubois and Prade (1980), we denote a classical set of the object $X$ called the universe, the generic elements of which are marked $x$. The membership of a classical subset $A$ of $X$ can be viewed as a membership (or characteristic) function from $X$ to $\{0,1\}$ such that

$$
\mu_{A}(x)=\left\{\begin{array}{ll}
1 & x \in A \\
0 & x \notin A
\end{array} .\right.
$$

The set $\{0,1\}$ is called a valuation set. In a more general case, the valuation set can also be stated as the 
real interval $\langle 0,1\rangle$. Then $A$ is called a fuzzy set (Zadeh, 1965). The value $\mu_{A}(x)$ characterizes the grade of membership of $x$ in $A$. The closer the value of $\mu_{A}(x)$ is to 1 , the more $x$ belongs to $A$. The fuzzy set $A$ may be written by the set of pairs as follows:

$$
A=\left\{\left(x, \mu_{A}(x)\right), x \in X\right\} .
$$

We can say that $A$ is a subset of $X$ that has no sharp boundaries.

Now let us introduce two basic operations with fuzzy sets - intersection and union - by Pedrycz et al. (2010). Two fuzzy sets $A, B$ and their membership functions $\mu_{A}, \mu_{B}$ are specified. The membership function of their intersection $A \cap B$ is computed in the form

$$
\mu_{A \cap B}(x)=\min \left(\mu_{A}(x), \mu_{B}(x)\right) \quad x \in X .
$$

The membership function of the union $A \cup B$ is determined as follows:

$$
\mu_{A \cup B}(x)=\max \left(\mu_{A}(x), \mu_{B}(x)\right) \quad x \in X .
$$

\section{2 (Triangular) fuzzy number}

A fuzzy number is a convex fuzzy set of the real line $R$ such that (Dubois and Prade, 1980)

a) $\exists ! x_{0} \in R, \mu_{A}\left(x_{0}\right)=1$ (is called the mean value of $A$ ),

b) $\mu_{A}(x)$ is piecewise continuous.

A fuzzy number intuitively represents a value that is inaccurate. This value can be characterized as about $x_{0}$. It belongs to a very frequent phenomenon in practice.

Novák (2000) alludes to the triangular fuzzy number as the most-used type of fuzzy number. Its membership function has the shape of a triangle, as we can see in the following graph (Figure 1).

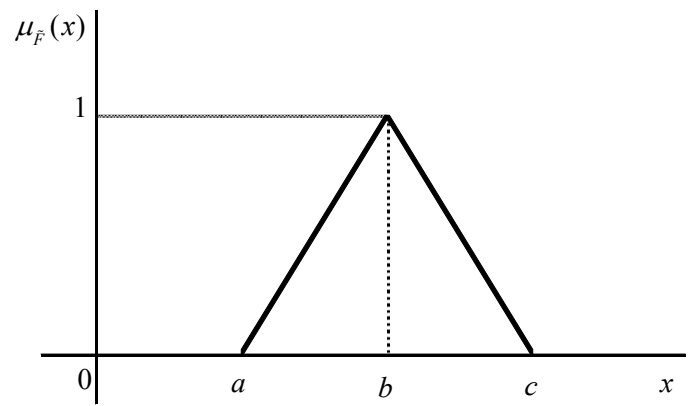

Figure 1 Membership function of a triangular fuzzy number

The membership function of the triangular fuzzy number $\tilde{F}$ is formalized as

$$
\mu_{\tilde{F}}(x)=\left\{\begin{array}{cc}
0 & x<a \wedge x>c \\
\frac{x-a}{b-a} & a \leq x \leq b \\
\frac{c-x}{c-b} & b \leq x \leq c \\
1 & x=b
\end{array},\right.
$$

where $a, b, c$ are parameters illustrated in the figure above. Concretely, $a$ can be interpreted as the lower bound, $b$ the peak point and $c$ the upper bound of the fuzzy number. Mostly the position of parameters $a, c$ is symmetric around the value of $b$. This means that the membership function usually creates an isosceles triangle. Of course, the different cases of the dissimilar sides of the triangle can be defined.

The triangular fuzzy number can be formally written as follows:

$$
\tilde{F}=(a, b, c) .
$$

Sometimes, it is necessary to keep available only left or right triangular fuzzy numbers. The membership function of the left triangular fuzzy number $\tilde{F}_{l}$ may be written as follows (Gupta and Bhattacharjee, 2010):

$$
\mu_{\tilde{F}_{l}}(x)=\left\{\begin{array}{cc}
0 & x<a, x>p \\
\frac{x-a}{b-a} & a \leq x \leq b, \\
1 & b \leq x \leq p
\end{array},\right.
$$

and for the right triangular fuzzy number $\tilde{F}_{r}$

$$
\mu_{\tilde{F}_{r}}(x)=\left\{\begin{array}{cc}
0 & x<0, x>c \\
\frac{c-x}{c-b} & b \leq x \leq c . \\
1 & 0 \leq x \leq b
\end{array} .\right.
$$

The membership function of the left and the right triangular fuzzy number is shown in Figure 2 and Figure 3.

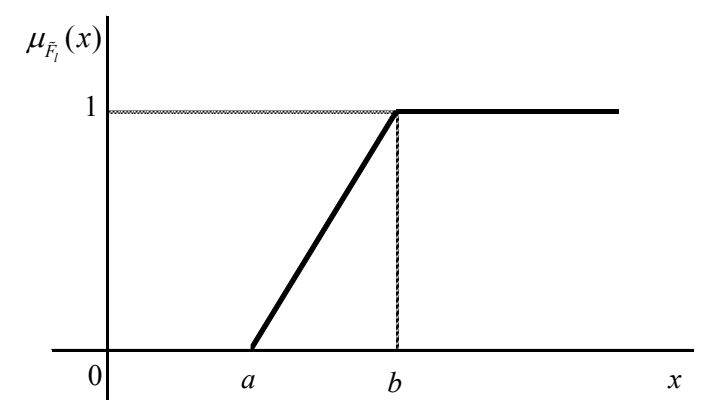

Figure 2 Membership function of the left triangular fuzzy number

The left or right triangular fuzzy number can be formally written as follows:

$$
\tilde{F}_{l}=(a, b, b) \quad \tilde{F}_{r}=(b, b, c) .
$$




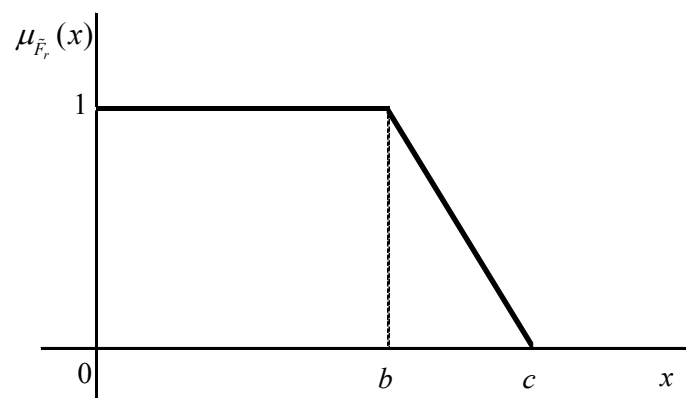

Figure 3 Membership function of the right triangular fuzzy number

One important question must be answered. Why is the triangular fuzzy number used? It is one of the favourite types of fuzzy numbers. Its membership function is piecewise continuous linear, so its computational operations are known and simpler. This type of membership function is able to express or approximate the decision maker's vague linguistic preferences.

\subsection{Linguistic variables}

A linguistic variable is a very important notion that is often used in practical applications. In general, a linguistic variable has values that are words and the meanings of these words are fuzzy sets in a certain universe. The values of a linguistic variable are called terms. Typical examples of this type of variable could be age, height, intelligence, etc. Their values could be young, high, clever, etc. The whole concept of linguistic variables was proposed by L.A. Zadeh (1975a, b, c).

Zimmermann (1991) characterizes a linguistic variable by a quintuple:

$$
(X, T(X), U, G, M),
$$

where $X$ is the name of the variable, $T(X)$ denotes the term set of $X$, that is, the set of names of linguistic values of $X, U$ is called the universum, $G$ is a syntactic rule for generating the value of $x \in T(X)$ from the set $T(X)$ and $M$ is the semantic rule for associating each term $x$ with its meaning $\tilde{M}(x) \tilde{\subset} U$.

$M$ assigns its meaning to each linguistic expression, which is a fuzzy set on a particular points scale, because psychological determination of the human temperament is not unambiguous. Therefore, it is better to define linguistic expressions as fuzzy sets than by means of a particular points scale, as occurred in the past (Novák, 1986). Each term $x$ is actually the name of a fuzzy variable $\langle x, U, R(x)\rangle$, the range of which is the set $R(x)=\tilde{M}(x)$. It is obvious that $\tilde{M}(x)$ is a fuzzy subset of $U$.

At the end of this chapter, the transformation procedure of linguistic expressions into fuzzy numbers will be introduced. The construction of a conversion scale is discussed for example by Baas and Kwakernaak (1977), Bonissone (1982), Keere (1982) and Chen (1988). At the beginning of the process, the particular scale of fuzzy numbers is prepared. Each fuzzy number describes a concrete linguistic meaning. For instance, I propose a situation depicted in the following picture (Figure 4).

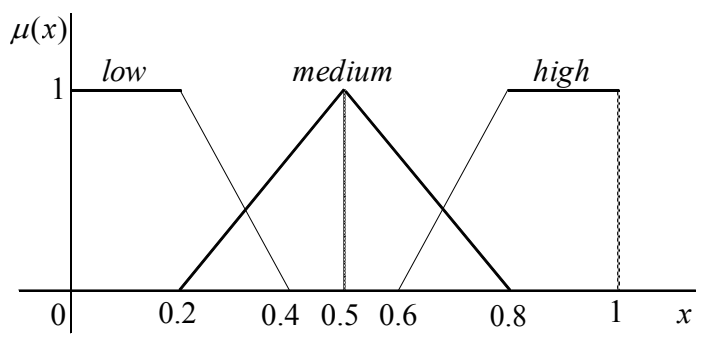

Figure 4 Scale of fuzzy numbers expressing linguistic meanings

In this illustrative case, we have a linguistic variable with three possible terms - low, medium and high. Therefore, we define three fuzzy numbers expressing these linguistic meanings. We could expect this example to describe the expression (attribute) of criterion relevance by a decision maker. Thus, the decision maker expresses the relevance of criteria linguistically and then these qualitative formulations are transformed into fuzzy numbers according to the mentioned scale in the interval from 0 to 1 .

Of course, the conversion scale can be more concrete (Chen and Hwang, 1992). For example, if the attribute is price, the set of terms can be extremely expensive, very expensive, ..., fair price, fairly cheap, ..., extremely cheap. For any type of attribute, we can always find a pair of words that represents extreme meanings, such as high vs. low, good vs. poor, etc. A set of 76 pairs of opposite words may be found in Osgood et al. (1975).

The scale of fuzzy numbers is constructed on the basis of a particular situation. Its construction could also be quite intuitive. It is possible to say that a detailed conversion scale is important when the decision maker is more familiar with the decisionmaking situation.

Some methodical approaches contain more conversion scales. Chen and Hwang (1992) show that when the number of conversion scales is greater, the system is able to cover more practical applications because it includes variously detailed scales (with 
different numbers of terms). The number of scales is discussed by Miller (1955) and Chen and Hwang (1992) in more detail.

\subsection{Bellman's approach to a fuzzy problem solution}

Bellman's approach is applied to the situation of the fuzzy multiple objective programming problem (Bellman and Zadeh, 1970).

We can consider the objective functions as the fuzzy goals. Further, in the model, there are fuzzy and strict constraints. Let us denote them. We have $k$ fuzzy goals $G_{j}(j=1,2, \ldots, k)$ and $m$ fuzzy constraints $C_{i}(i=1,2, \ldots, m)$. The strict constraints align the set of the solution feasibility $X$ :

$$
X=\left\{\mathbf{x} \in R^{n}, p_{i}(\mathbf{x}) R_{i} b_{i}, i=1,2, \ldots, m\right\},
$$

where $\mathbf{x}=\left(x_{1}, x_{2}, \ldots, x_{n}\right)$ is the vector of unknown variables, $p_{i}(\mathbf{x})$ is the left side (a function), $R_{i}$ is the relation mark and $b_{i}$ is the right side (a constant) of the $i$-th constraint. Fuzzy goals and constraints are actually the same; they have an analogical function. Each fuzzy goal has the membership function $\mu_{G_{j}}(\mathbf{x})$, and each fuzzy condition has the membership function $\mu_{C_{i}}(\mathbf{x})$.

The fuzzy solution is the fuzzy set that is created as an intersection of fuzzy goals and constraints. Regarding the solution feasibility set, the fuzzy decision is formulated as the following fuzzy set $A$ :

$$
A=G_{1} \cap G_{2} \cap \ldots \cap G_{k} \cap C_{1} \cap C_{2} \cap \ldots \cap C_{m} \cap X .
$$

Then this fuzzy set will have the membership function $\mu_{A}(\mathbf{x})$ as follows:

$$
\begin{aligned}
& \mu_{A}(\mathbf{x})=\mu_{G_{1}}(\mathbf{x}) \wedge \mu_{G_{2}}(\mathbf{x}) \wedge \ldots \wedge \mu_{G_{k}}(\mathbf{x}) \wedge \\
& \wedge \mu_{C_{1}}(\mathbf{x}) \wedge \mu_{C_{2}}(\mathbf{x}) \wedge \ldots \wedge \mu_{C_{m}}(\mathbf{x})= \\
& =\min _{i, j}\left\{\mu_{G_{j}}(\mathbf{x}), \mu_{C_{i}}(\mathbf{x})\right\} .
\end{aligned}
$$

As an illustration (Bellman, 1970), the fuzzy decision for a simplified situation of one fuzzy goal and one fuzzy constraint is displayed in the following picture (Figure 5).

A maximizing solution can be as follows:

$$
\mu_{A}\left(\mathbf{x}^{\mathbf{M}}\right)=\left\{\begin{array}{cc}
\max \mu_{A}(\mathbf{x}) & x \in X \\
0 & x \notin X
\end{array} .\right.
$$

If the function $\mu_{A}(\mathbf{x})$ has one unique maximum $\mathbf{x}^{\mathbf{M}}$, then this solution can be classified as strictly representing all the goals and limits with the highest grade of membership possible. It is obvious that the final result is calculated on the basis of the maximin operator.

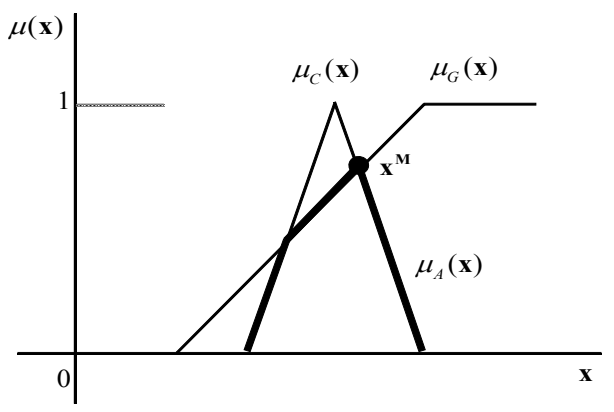

Figure 5 Genesis of the fuzzy solution

To find the maximum of the membership function $\mu_{A}(\mathbf{x})$ in terms of the problem with fuzzy goals and conditions, the supporting model is formulated by Zimmermann (1978):

$$
\begin{aligned}
& \lambda \rightarrow \max \\
& \mu_{G_{j}}(\mathbf{x}) \geq \lambda \quad j=1,2, \ldots, k \\
& \mu_{C_{i}}(\mathbf{x}) \geq \lambda \quad i=1,2, \ldots, m \\
& \mathbf{x} \in X \\
& \lambda \in\langle 0,1\rangle,
\end{aligned}
$$

where $\mu_{A}(\mathbf{x})=\min _{i, j}\left\{\mu_{G_{j}}(\mathbf{x}), \mu_{C_{i}}(\mathbf{x})\right\}$.

The described approach will be applied to the construction of crisp criteria weights.

\section{Fuzzy weight estimation method}

As mentioned above, a decision maker expresses the criterion relevance linguistically. This information is transformed into a conversion scale of fuzzy numbers prepared beforehand. Thus, some values from the interval $\langle 0,1\rangle$ with some tolerance zones are assigned to each linguistic term. Then, the $j$-th vague (fuzzy) weight is formulated as a triangular fuzzy number $\tilde{w}_{j}$

$$
\tilde{w}_{j}=\left(v_{j}-\delta_{j}^{l}, v_{j}, v_{j}+\delta_{j}^{u}\right),
$$

where $v_{j}(j=1,2, \ldots, k)$ is the weight corresponding most to the linguistic expression of criterion relevance and $\delta_{j}^{l}, \delta_{j}^{u}(j=1,2, \ldots, k)$ specify the tolerance zone for the weight value of the $j$-th criterion.

The membership function of such a defined criterion weight as the fuzzy set $\tilde{w}_{j}$ has the following form: 


$$
\mu_{\tilde{w}_{j}}\left(w_{j}\right)=\left\{\begin{array}{cc}
0 & w_{j}<v_{j}-\delta_{j}^{l} \\
\frac{w_{j}+\delta_{j}^{l}-v_{j}}{\delta_{j}^{l}} & v_{j}-\delta_{j}^{l} \leq w_{j} \leq v_{j} \\
\frac{\delta_{j}^{u}+v_{j}-w_{j}}{\delta_{j}^{u}} & v_{j} \leq w_{j} \leq v_{j}+\delta_{j}^{u} \\
0 & w_{j}>v_{j}+\delta_{j}^{u}
\end{array} .\right.
$$

The membership function can be graphically demonstrated as follows (Figure 6).

There is no doubt that the fuzzy number must not be defined symmetrically, so $\delta_{j}^{l} \neq \delta_{j}^{u}$ is possible.

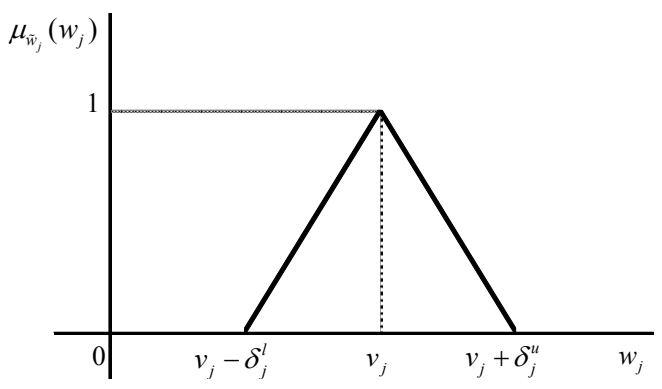

Figure 6 Membership function of fuzzy weight $\tilde{w}_{j}$

\subsection{Simple model}

In terms of the next step, the fuzzy mathematical model will be formulated in order to set the quantitative weights of all the criteria. We have $k$ fuzzy goals describing the criteria relevance and no fuzzy constraints. The weights are standardized, and the sum of them is one. The solution will give the crisp values of criteria weights with the greatest possible grade of membership of its membership function. The supporting model of linear programming computationally based on the maximin approach is defined as follows:

$$
\begin{aligned}
& z=\alpha \rightarrow \max \\
& \frac{w_{j}+\delta_{j}^{l}-v_{j}}{\delta_{j}^{l}} \geq \alpha \quad j=1,2, \ldots, k \\
& \frac{\delta_{j}^{u}+v_{j}-w_{j}}{\delta_{j}^{u}} \geq \alpha \quad j=1,2, \ldots, k \\
& \sum_{j=1}^{k} w_{j}=1 \\
& w_{j} \geq 0 \quad j=1,2, \ldots, k \\
& \alpha \in\langle 0,1\rangle,
\end{aligned}
$$

where $\alpha=\min _{j}\left[\mu_{\tilde{w}_{j}}\left(w_{j}\right)\right]$ is the membership function of a fuzzy set representing the final fuzzy decision. This set is formulated as an intersection of all the fuzzy and strict sets and has a unique maximum. In order to gain the maximal grade of the solution's membership, the objective function is maximizing.
The crisp weight value of the $j$-th criterion is denoted by $w_{j}$.

Let us warn readers of some problems of this model specification in the crisp weight determination procedure. Imagine the situations

$$
\sum_{j=1}^{k}\left(v_{j}+\delta_{j}^{u}\right)<1 \text {, or } \sum_{j=1}^{k}\left(v_{j}-\delta_{j}^{l}\right)>1 .
$$

It is obvious that the solution is not feasible. The values of weights cannot be chosen from the interval $\left\langle v_{j}-\delta_{j}^{l}, v_{j}, v_{j}+\delta_{j}^{u}\right\rangle$. To eliminate this shortcoming, some modifications can be made. Firstly, the conversion scale can be modified for the concrete situation, thus for the particular number of criteria and the expressed criteria relevance. However, this method is a little misleading, because then we can set the criteria weights straight without any procedure. It is natural that the conversion scale is made before the expressed criteria relevance. Another method is to modify the condition $\alpha \in\langle 0,1\rangle$ to $\alpha \leq 1$. Then values outside the interval $\left\langle v_{j}-\delta_{j}^{l}, v_{j}, v_{j}+\delta_{j}^{u}\right\rangle$ are possible, so the limit $\sum_{j=1}^{k} w_{j}=1$ can be accepted. On the other hand, now the value $\alpha$ has no real economic interpretation because the grade of membership is negative on the basis of the model formulation. However, it is obvious that the actual value of membership of the solution is zero. To solve this problem, a more comprehensive model will be formulated.

\subsection{Comprehensive model}

The following model will be expressed in parts in order to comprehend the whole principle more clearly. The first part of the model focuses on the left side of the triangular fuzzy numbers. Let us assume the inequalities

$$
\begin{aligned}
& w_{j} \leq v_{j}-\delta_{j}^{l}+M\left(1-p_{j}\right) \quad j=1,2, \ldots, k \\
& \alpha \leq 0+M\left(1-p_{j}\right) \quad j=1,2, \ldots, k \\
& w_{j} \leq v_{j}+M\left(1-q_{j}\right) \quad j=1,2, \ldots, k \\
& w_{j} \geq v_{j}-\delta_{j}^{l}-M\left(1-q_{j}\right) \quad j=1,2, \ldots, k \\
& \frac{w_{j}+\delta_{j}^{l}-v_{j}}{\delta_{j}^{l}} \geq \alpha-M\left(1-q_{j}\right) \quad j=1,2, \ldots, k \\
& p_{j}+q_{j} \leq 1 \quad j=1,2, \ldots, k \\
& p_{j}, q_{j} \in\{0,1\}, M=10^{3},
\end{aligned}
$$

where $w_{j}, v_{j}, \delta_{j}^{l}(j=1,2, \ldots, k)$ and $\alpha$ symbolize the same variables as in the previous model (1). The first two conditions describe the situation when the weight 
value is lower than (or equal to) the lower bound of interval $\left\langle v_{j}-\delta_{j}^{l}, v_{j}\right\rangle$, then the grade of the membership of the final solution will be zero. The next three constraints are included in the modified model to ensure that the value of the weight will be in the interval $\left\langle v_{j}-\delta_{j}^{l}, v_{j}\right\rangle$. The big $M$ ensures that the appropriate constraints subside to be limits in the model if the binary variables are 0 . The condition $p_{j}+q_{j} \leq 1 \quad(j=1,2, \ldots, k)$ determines that only one or none of the mentioned situations can occur.

The second part of the model focuses on the right side of the triangular fuzzy numbers. It is formulated as follows:

$$
\begin{aligned}
& w_{j} \geq v_{j}+\delta_{j}^{u}-M\left(1-r_{j}\right) \quad j=1,2, \ldots, k \\
& \alpha \leq 0+M\left(1-r_{j}\right) \quad j=1,2, \ldots, k \\
& w_{j} \geq v_{j}-M\left(1-s_{j}\right) \quad j=1,2, \ldots, k \\
& w_{j} \leq v_{j}+\delta_{j}^{u}+M\left(1-s_{j}\right) \quad j=1,2, \ldots, k \\
& \frac{\delta_{j}^{u}+v_{j}-w_{j}}{\delta_{j}^{u}} \geq \alpha-M\left(1-s_{j}\right) \quad j=1,2, \ldots, k \\
& r_{j}+s_{j} \leq 1 \quad j=1,2, \ldots, k \\
& r_{j}, s_{j} \in\{0,1\}, M=10^{3},
\end{aligned}
$$

where $w_{j}, v_{j}, \delta_{j}^{u}(j=1,2, \ldots, k)$ and $\alpha$ symbolize the same variables as in models (1) and (3). The first two conditions describe the situation when the weight value is greater than (or equal to) the upper bound of the interval $\left\langle v_{j}+\delta_{j}^{u}, v_{j}\right\rangle$, then the grade of the membership of the final solution will be zero. The next three constraints ensure that the value of the weight is in the interval $\left\langle v_{j}+\delta_{j}^{u}, v_{j}\right\rangle$. The other conditions and elements are the same as in (4).

Finally, the following conditions are added to the model:

$$
\begin{aligned}
& p_{j}+q_{j}+r_{j}+s_{j}=1 \quad j=1,2, \ldots, k \\
& \sum_{j=1}^{k} w_{j}=1 \\
& w_{j} \geq 0 \quad j=1,2, \ldots, k \\
& \alpha \in\langle 0,1\rangle .
\end{aligned}
$$

Because only one of the four situations regarding the fuzzy number value described above can occur, the sum of all the binary variables must be one. Of course, the conditions of weight standardization, nonnegativity and binary conditions are also formulated.
The whole model is constructed as the union of the fractional parts (3), (4) and (5) with the added objective function $\alpha$.

$$
\begin{aligned}
& z=\alpha \rightarrow \max \\
& w_{j} \leq v_{j}-\delta_{j}^{l}+M\left(1-p_{j}\right) \quad j=1,2, \ldots, k \\
& \alpha \leq 0+M\left(1-p_{j}\right) \quad j=1,2, \ldots, k \\
& w_{j} \leq v_{j}+M\left(1-q_{j}\right) \quad j=1,2, \ldots, k \\
& w_{j} \geq v_{j}-\delta_{j}^{l}-M\left(1-q_{j}\right) \quad j=1,2, \ldots, k \\
& \frac{w_{j}+\delta_{j}^{l}-v_{j}}{\delta_{j}^{l}} \geq \alpha-M\left(1-q_{j}\right) \quad j=1,2, \ldots, k \\
& p_{j}+q_{j} \leq 1 \quad j=1,2, \ldots, k \\
& w_{j} \geq v_{j}+\delta_{j}^{u}-M\left(1-r_{j}\right) \quad j=1,2, \ldots, k \\
& \alpha \leq 0+M\left(1-r_{j}\right) \quad j=1,2, \ldots, k \\
& w_{j} \geq v_{j}-M\left(1-s_{j}\right) \quad j=1,2, \ldots, k \\
& w_{j} \leq v_{j}+\delta_{j}^{u}+M\left(1-s_{j}\right) \quad j=1,2, \ldots, k \\
& \frac{\delta_{j}^{u}+v_{j}-w_{j}}{\delta_{j}^{u}} \geq \alpha-M\left(1-s_{j}\right) \quad j=1,2, \ldots, k \\
& r_{j}+s_{j} \leq 1 \quad j=1,2, \ldots, k \\
& p_{j}+q_{j}+r_{j}+s_{j}=1 \quad j=1,2, \ldots, k \\
& \sum_{j=1}^{k} w_{j}=1 \\
& w_{j} \geq 0 \quad j=1,2, \ldots, k \\
& \alpha \in\langle 0,1\rangle, M=10^{8} \\
& p_{j}, q_{j}, r_{j}, s_{j} \in\{0,1\} \quad j=1,2, \ldots, k .
\end{aligned}
$$

It is clear that the presence of constraint

$$
p_{j}+q_{j}+r_{j}+s_{j}=1 \quad j=1,2, \ldots, k
$$

enables us to eliminate the following two conditions:

$$
p_{j}+q_{j} \leq 1 \quad j=1,2, \ldots, k, \quad r_{j}+s_{j} \leq 1 \quad j=1,2, \ldots, k .
$$

Accordingly, the model becomes simpler because $2 k$ constraints are removed.

Model (6) embodies some weakness in the situation (2). The value of the objective function is evaluated correctly; however, the final weights of some criteria may be very different from their given interval. It is possible that some weights can be much greater than $v_{j}+\delta_{j}^{u}$ or much lower than $v_{j}-\delta_{j}^{l}$. Then the result must not make sense in terms of human intuition.

The problem is that we gain an infinite number of alternative solutions with the objective function equal to zero. This situation does not occur in model (1) with the modified condition $\alpha \leq 1$. Because the 
objective function is negative as rarely as possible, the deviations of the weight values from the given interval are limited.

To solve the problem described in model (6), some other conditions for weight values will be added. In the case of $\sum_{j=1}^{k}\left(v_{j}+\delta_{j}^{u}\right)<1$, the following constraint will be formulated:

$$
w_{j} \leq v_{j}+\delta_{j}^{u}+\Delta_{j}^{u} \quad j=1,2, \ldots, k,
$$

and for the situation $\sum_{j=1}^{k}\left(v_{j}-\delta_{j}^{d}\right)>1$

$$
v_{j}-\delta_{j}^{l}-\Delta_{j}^{l} \leq w_{j} \quad j=1,2, \ldots, k,
$$

where $\Delta_{j}^{l}, \Delta_{j}^{u}$ extend the tolerance zone for the weight of the $j$-th criterion. The main question is how to determine these values. It is mainly up to human intuition, but there is no doubt that we want the deviations from the original interval to be as small as possible. The extension should be made proportionally for all the criteria in order to respect the original mutual location of the fuzzy numbers. We proposed the technique when the particular interval of the fuzzy number is extended by the percentage part of the left or right side of the fuzzy number. It is possible that the additional tolerance zones will be insufficient to gain the solution, in which case the intervals must be extended. This fact is easily legible before a modification process.

The comprehensive model becomes computationally more complicated than model (1) because of the greater number of constraints and the binary variables, but for the situation of a smaller number of criteria (tens), it is not a problem to solve it in an acceptable amount of time.

\subsection{Procedure for the usage of the described method}

In conclusion, let us think about the usage of the described models. First, we consider model (1). If the model has a feasible solution, we have the quantitative values of the criteria weights. If this model has no solution, situation (2) occurs. Of course, the nonfeasibility of the solution can also be identified according to the computation of the sum of the lower or upper bounds of all the fuzzy numbers. Now two methods are possible.

We can modify model (1) via the condition $\alpha \leq 1$. This model provides the optimal solution with a negative value of the objective function. It is not possible to interpret this fact correctly in terms of the concept of the grade of the membership in the fuzzy set theory. However, there is no doubt that the solution is applicable to the practical decision-making situation.

The second method is to use model (6) with the additional condition (7) or (8) according to the particular situation. The disadvantage of the model is the fact that additional conditions for the weights are required and alternative solutions exist. On the other hand, a broader scale of solutions can offer a wider view of the decision-making situation.

Finally, we do not want to say which approach is better or worse. Simply, two alternative models are available and can be applied separately.

The weight estimation procedure will be summarized via the following steps:

Step 1: We have concrete evaluative criteria. The importance of the criteria is expressed linguistically by the decision maker.

Step 2: The conversion scale of linguistic expressions to fuzzy numbers is constructed. The supporting model (1) for the fuzzy programming problem is formulated in order to quantify the weights. If the solution is found, the algorithm results. If not, go to the next step.

Step 3: We can apply the modified version of model (1) with the condition $\alpha \leq 1$. Alternatively, the particular case of (2) is identified and then model (6) with (7) or (8) is employed.

Step 4: We receive the crisp values of the weights.

\section{Practical application}

Consider the situation in which a potential investor is deciding about the investment in the open share funds offered and managed by Česká spořitelna Investment Company. Three groups of share funds are available mixed, bond and stock funds. The investor wants to choose only one fund from each group to share in his investment portfolio. The whole decision-making procedure is described for example by Borovička (2012) or Borovička (2013), but now the fractional part of the criteria weight estimation is our interest. The criteria will be return, risk and costs.

We will illustrate a few cases and solve them by the approaches described above.

Firstly, we define the linguistic variable in the mentioned structure $(X, T(X), U, G, M)$. The name of the variable $X$ can be criterion relevance, then

$$
X=\text { criterion relevance. }
$$

The potential investor evaluates the criteria importance on the simple scale with three terms - low, medium, high. Thus, the set $T(X)$ will be formulated as follows: 


$$
T(X)=\{\text { low, medium, high }\} .
$$

It is possible to define the universe $U$ as the set $T(X)$ or it can be defined in a wider range of linguistic terms expressing the criterion importance in which our three terms will be included. The syntactic rule $G$ is only reduced to the enumeration of the mentioned linguistic terms. The semantic rule $M$ assigns a meaning to each word, which is a fuzzy set on the given scale. The semantic rule will be differentiated according to the particular situation described below.

All the mathematical models will be solved in the LINGO optimization software.

\subsection{Situation 1}

The structure of fuzzy evaluations and the linguistic expressions of criterion relevance are presented in the following table (Table 1). The structure is subjectively formulated.

Table 1 Situation 1

\begin{tabular}{|l|c|c|}
\hline Criterion & Relevance & Fuzzy number \\
\hline Costs & Low & $\langle 0.05,0.05,0.20\rangle$ \\
\hline Return & Medium & $\langle 0.20,0.25,0.30\rangle$ \\
\hline Risk & High & $\langle 0.50,0.70,0.70\rangle$ \\
\hline
\end{tabular}

The sum of the peak points of all the fuzzy numbers is one, so the solution is expectable. The weights just equal those values according to both model (1) and model (6) with the objective function $z=\alpha=1$. In this case, the usage of model (6) is not necessary.

For any other case when both conditions (2) do not hold, both models provide the same solution with an adequate value of the objective function $\alpha \in\langle 0,1\rangle$.

\subsection{Situation 2}

The second situation describes the fact that one of the conditions (2) holds. For instance, we have the following case (Table 2). The structure is subjectively formulated.

Table 2 Situation 2

\begin{tabular}{|l|c|c|}
\hline Criterion & Relevance & Fuzzy number \\
\hline Costs & Very low & $\langle 0.10,0.15,0.20\rangle$ \\
\hline Return & Low & $\langle 0.10,0.20,0.30\rangle$ \\
\hline Risk & Medium & $\langle 0.10,0.35,0.40\rangle$ \\
\hline
\end{tabular}

It is obvious that $\sum_{j=1}^{k}\left(v_{j}+\delta_{j}^{u}\right)<1$ holds. Then the original model (1) has no feasible solution. Model (1) with the modified condition $\alpha \leq 1$ is applied. We gain the solution with a negative value of the objective function, but we know that the real membership grade of the solution is zero. The final weights are shown in the following table (Table 3).

Table 3 Criteria weights by modified model (1)

\begin{tabular}{|l|c|}
\hline Criterion & Weight \\
\hline Costs & 0.225 \\
\hline Return & 0.350 \\
\hline Risk & 0.425 \\
\hline
\end{tabular}

If the comprehensive model (6) is employed, the solution will be found with a zero objective function (Table 4).

Table 4 Criteria weights by comprehensive model (1)

\begin{tabular}{|l|c|}
\hline Criterion & Weight \\
\hline Costs & 0.150 \\
\hline Return & 0.500 \\
\hline Risk & 0.350 \\
\hline
\end{tabular}

As we can see in the table, the second most important criterion receives the greatest weight, which is against human intuition. In order to gain a more real solution, model (6) will be modified by the additional constraint (7). Consequently, the upper bounds will be increased by $50 \%{ }^{1}$ of the right interval length of each fuzzy number. Then it must hold (Table 5).

Table 5 Additional conditions for the weights

\begin{tabular}{|l|c|}
\hline Criterion & Weight \\
\hline Costs & weight $\geq 0.225$ \\
\hline Return & weight $\geq 0.350$ \\
\hline Risk & weight $\geq 0.425$ \\
\hline
\end{tabular}

The result of the modified model (6) is presented in the following table (Table 6).

Table 6 Criteria weights by modified comprehensive model (6)

\begin{tabular}{|l|c|}
\hline Criterion & Weight \\
\hline Costs & 0.225 \\
\hline Return & 0.350 \\
\hline Risk & 0.425 \\
\hline
\end{tabular}

As we can see, the modification of the upper bounds is made so that the sum of them is one. The result is the same as in the application of modified model (1). If the increasing upper bounds were greater, a different solution would be found. In comparison with the original model (6), the weights correspond to the fuzzy evaluation much better. The objective function equals zero.

\footnotetext{
${ }^{1}$ This is the minimum to solve the problem. Of course, the increasing upper bounds could be greater.
} 


\subsection{Result discussion}

These two situations were selected as rather illustrative to understand the principle of the proposed methods. Of course, they depend on the conversion scale. It can have such a form that excludes the cases (2). It is influenced by the parameters of the triangular fuzzy number as well as the number of criteria. The conversion scale can be adapted to the particular decision-making situation. Then the simple model or other mentioned methods can be applied.

The proposed methods can be applied to any multiple-criteria decision-making situation, in which the criteria relevance is expressed linguistically and it is necessary to transform it into a crisp form (weights), for example in the capital market, banking or manufacturing. It is obvious that the described procedure can be part of many multiple-criteria decision-making methods.

\subsection{Comparison with other methods}

The proposed method is presented as an alternative to the current approaches with its advantages and disadvantages.

We have a group of methods using defuzzification formulae (Chen et al., 1992). The transformation procedure can be based on the index of optimism (Lee, 1999), which is used in fuzzy AHP (Cheng et al., 1994). In the transformation process, the concept $\alpha$-level is often applied (e.g. Detyniecki et al., 2001). Many defuzzification formulae are proposed. Evidently, these formulae cannot ensure normalization of the weights. Consequently, in the next step, normalization must be carried out. The advantage of the proposed method is that the normalization is included in the model. On the other side, the computation process can be longer. Other methods use the composite membership function in order to express fuzzy priorities (Narasimhan, 1981). Then the crisp mathematical model is formulated in order to set the crisp weights. This process is usually computationally more difficult and it can be less comprehensible for a user.

Finally, the new fuzzy weights estimation method is proposed as another approach to set the crisp weights of criteria. The procedure is comprehensible for the user and not too computationally difficult. It is based on the optimization process that tries to gain a grade of membership for each weight that is as great as possible.

\section{Conclusion}

The main aim of this article is to introduce the criteria weight estimation procedure on the basis of linguistic expressions about the criteria relevance from the decision maker. For this purpose, the concepts of fuzzy sets, fuzzy numbers and linguistic variables are used. The linguistic meanings are transformed into triangular fuzzy numbers, then the proposed mathematical models of linear programming based on the maximin principle are solved in order to find the final quantitative values of weights. We point out the shortcomings of the described models and make appropriate modifications.

The quantitative weights of the evaluative criteria are further used in the whole multiple-criteria decision-making procedure in terms of many possible multiple-criteria decision-making methods.

The proposed approaches are applied to a real decision-making situation in the capital market with open share funds in order to demonstrate the possible situations and difficulties in the whole procedure.

\section{Acknowledgements}

The research project was supported by Grant No. IGA F4/19/2013 of the Internal Grant Agency, Faculty of Informatics and Statistics, University of Economics, Prague and Grant No. 13-07350S of the Grant Agency of the Czech Republic.

\section{References}

BAAS, S.M., KWAKERNAAK, H. (1977). Rating and ranking of multiple aspect alternative using fuzzy sets. Automatica 13(1): 47-58. http://dx.doi.org/10.1016/0005-1098(77)90008-5

BELLMAN, R.E., ZADEH, L.A. (1970). Decisionmaking in a fuzzy environment. Management Science 17(4): 141-164.

BONISSONE, P.P. (1982). A fuzzy sets based linguistic approach: Theory and applications. In: Gupta, M.M., Sanchez, E. (eds.): Approximate Reasoning in Decision Analysis. Amsterdam: North-Holland, 329339.

BOROVIČKA, A. (2013). Metodický aparát k vytváření portfolia otevřených podílových fondů. In: Védecká konference doktorského studia FIS 2013, Praha: University of Economics, 118-133.

BOROVIČKA, A. (2012). The investment decision making under uncertainty. In: Mathematical methods in economics 2012. Opava: Silesian University in Opava, 37-42.

BROŽOVÁ, H., HOUŠKA, M., ŠUBRT, T. (2009). Modely pro vícekriteriální rozhodování. Praha: ČZU.

CHEN, S.J., HWANG, C.L. (1992). Fuzzy Multiple Attribute Decision Making: Methods and Applications. Berlin: Springer-Verlag. http://dx.doi.org/10.1007/978-3-642-46768-4 
CHEN, S.M. (1988). A new approach to handling fuzzy decision-making problems. In: 18th International Symposium on Multiple-Valued Logic. Palma de Mallorca: UIB, 72-76.

CHENG, C. H., MON, D. L. (1994). Evaluating weapon system by analytical hierarchy process based on fuzzy scales. Fuzzy Sets and Systems 63: 1-10.

DETYNIECKI, M., YAGER, R. R. (2001). Ranking fuzzy numbers using $\alpha$-weighted valuations. Fuzziness and Knowledge Based Systems 8: 573-592.

DUBOIS, D., PRADE, H. (1980). Fuzzy Sets and Systems: Theory and Applications. New York: Academic Press.

FIALA, P. (2008). Modely a metody rozhodování. Praha: Oeconomica.

GUPTA, M., BHATTACHARJEE, D. (2010). Multi objective problem in fuzzy environment where resources are triangular fuzzy number. European Journal of Scientific Research 46(1): 99-106.

HWANG, C.L., YOON, K. (1981). Multiple Attribute Decision Making - Methods and Applications, a Stateof-the-Art Survey. New York: Springer-Verlag. http://dx.doi.org/10.1007/978-3-642-48318-9

KERRE, E.E. (1982). The use of fuzzy set theory in electrocardiological diagnostics. In: Gupta, M.M., Sanchez, E. (eds.): Approximate Reasoning in Decision Analysis. Amsterdam: North-Holland, 277-282.

LAI, Y.J., HWANG, C.L. (1996). Fuzzy Multiple Objective Decision Making: Methods and Applications. $2^{\text {nd }}$ corrected ed. Berlin: Springer-Verlag.

LEE, A. R. (1999). Application of Modified Fuzzy AHP Method to Analyze Bolting Sequence to Structural Joints. USA: Bell \& Howell Company.

MILLER, G.A. (1955). The magic number seven, plus or minus seven. Psychological Review 101(2): 343352. http://dx.doi.org/10.1037/0033-295X.101.2.343

NARASIMHAN, R. (1981). On fuzzy goal programming - some comments. Decision Science 12(3): 532538. http://dx.doi.org/10.1111/j.1540-5915.1981.tb00103.x NOVÁK, V. (2000). Základy fuzzy modelování. Praha: BEN.

NOVÁK, V. (1986). Fuzzy množiny a jejich aplikace. Praha: SNTL.

OSGOOD, C.E., SUCI, G.J., TENNENBAUM, P.H. (1975). The Measurement of Meaning. Urbana: University of Illinois Press.

PEDRYCZ, W., EKEL, P., PARREIRAS, R. (2010). Fuzzy Multicriteria Decision-Making: Models, Methods and Applications. New Jersey: Wiley.

ZADEH, L.A. (1975a). The concept of a linguistic variable and its application to approximate reasoning I. Information Sciences 8(3): 199-249.

http://dx.doi.org/10.1016/0020-0255(75)90036-5

ZADEH, L.A. (1975b). The concept of a linguistic variable and its application to approximate reasoning II. Information Sciences 8(4): 301-357.

http://dx.doi.org/10.1016/0020-0255(75)90046-8

ZADEH, L.A. (1975c). The concept of a linguistic variable and its application to approximate reasoning III. Information Sciences 9(1): 43-80.

http://dx.doi.org/10.1016/0020-0255(75)90017-1

ZADEH, L.A. (1965). Fuzzy sets. Information and Control 8(3): 338-353.

http://dx.doi.org/10.1016/S0019-9958(65)90241-X

ZIMMERMANN, H.J. (1991). Fuzzy Set Theory and its Applications. Boston: Kluwer academic Publishers. http://dx.doi.org/10.1007/978-94-015-7949-0

ZIMMERMANN, H.J. (1978). Fuzzy programming and linear programming with several objective functions. Fuzzy Sets and Systems 1(1): 45-55.

http://dx.doi.org/10.1016/0165-0114(78)90031-3 